Research Article

\title{
Impact of Additives on Heterogeneous Crystallization of Acetaminophen
}

\author{
Hsinyun Hsu, ${ }^{1}$ Lynne S. Taylor, ${ }^{2}$ and Michael T. Harris $\mathbb{i D}^{1}$ \\ ${ }^{1}$ Department of Chemical Engineering, Purdue University, 480 Stadium Mall Drive, West Lafayette, IN 47907, USA \\ ${ }^{2}$ Department of Industrial and Physical Pharmacy, Purdue University, 575 Stadium Mall Drive, West Lafayette, IN 47907, USA
}

Correspondence should be addressed to Michael T. Harris; mtharris@purdue.edu

Received 4 May 2018; Accepted 27 September 2018; Published 11 November 2018

Academic Editor: M. G. Ierapetritou

Copyright (C) 2018 Hsinyun Hsu et al. This is an open access article distributed under the Creative Commons Attribution License, which permits unrestricted use, distribution, and reproduction in any medium, provided the original work is properly cited.

Introducing foreign substrates or additives is the common way to regulate polymorphism or kinetics of crystallization. Most present studies consider the substrate factor and additive factor separately. Here, the interplay between the additive, crystallizing molecules, and the substrate was investigated. Acetaminophen (APAP) was used as the model compound. 5 wt.\% dioctyl sodium sulfosuccinate (AOT), poly(acrylic) acid (PAA), and hydroxypropyl methylcellulose (HPMC) were employed as additives. The interfacial crystal growth rate of APAP in the presence of additives was studied between slides coated with chitosan (CS) film. The crystallization kinetics of the additive/APAP mixture on CS substrate was also investigated. The additive/APAP was characterized by differential scanning calorimetry (DSC), and the interfacial molecular interaction was studied by Fourier transform infrared spectroscopy (FTIR). The results indicate that the additive-substrate interaction can change the interfacial growth behavior observed in the additive-APAP binary system. Nevertheless, crystallizing without confinement, the additive-APAP interaction is more effective at controlling the crystallization of APAP, and the substrates did not have much effect.

\section{Introduction}

Controlling crystallization is important in many areas of science and technology, such as manufacturing of pharmaceuticals, production of semiconductors and nonlinear optics, as well as the formation of biominerals. To obtain crystals with the desired structure and properties, foreign substrates or additives are often introduced.

In most practical circumstances, crystallization begins with heterogeneous nucleation on foreign substrates [1]. Therefore, foreign surfaces can be used to dictate crystallization of molecules and form crystals with the desired properties. Carters et al. [2] had shown that crystalline substrates which are lattice-matched with particular crystal faces have been used for direct nucleation of organic crystals and even control polymorph selectivity by epitaxy. Studies discussing the effects of porous substrates with pores that have various shapes and spherical aggregation on heterogeneous nucleation have been done by Diao et al. [3] and Quon et al. [4], and their results demonstrate the crucial impact of surface morphology on crystallization. Additionally, although the mechanism is not clear, different polymorphs of compounds have been successfully formed on amorphous polymeric films. Examples of small molecules [5], supramolecular complexes [5], and proteins [6] had been shown.

In addition to substrates, introducing additives is another popular approach to control crystallization. Tailormade auxiliaries that can have molecular recognition with crystallizing molecules have been used to change crystallization behavior [7]. Generally, the tailor-made auxiliaries are classified into two categories: inhibitors and promoters. A variety of purposes including morphology control [8], assignment of the absolute structure of chiral molecules and polar crystals [9], elucidation of the impact of solvent on crystal growth [10], and crystallization of desired polymorph [11] can be achieved by using the inhibitor. As for the promoter, monolayers of amphiphilic molecules on water have been employed to induce the growth of a variety of three-dimensional crystals at the monolayer-solution interface by structural match [12], molecular complementarity [13], or electrostatic interaction [14]. In pharmaceutical sciences, compatible polymers usually serve as additives to 
stabilize the amorphous state of poorly water-soluble active pharmaceutical ingredients (APIs) and increase their bioavailability. Polymers are thought to inhibit crystallization of the amorphous solid by increasing the glass transition temperature $\left(T_{\mathrm{g}}\right)$ of the resultant molecular level blend (often termed an amorphous solid dispersion), leading to a decrease in mobility of the API molecules, and through the formation of API-polymer specific interactions which act to disrupt self-assembly [15].

Given the influence of substrates [16] and additives, exclusively, it would be interesting to investigate their combinational effects on crystallization. In this study, crystallization of acetaminophen (APAP) on chitosan (CS) substrates [16] in the presence of additives was examined. The impact of additives on crystallization of APAP on the citric CS film was compared. Dioctyl sodium sulfosuccinate (AOT), polyacrylic acid (PAA), and hydroxypropyl methylcellulose (HPMC), which have various extents of molecular interaction with APAP, were used as the additives. The interplay between APAP, additives, and substrates was studied.

\section{Materials and Methods}

2.1. Materials. Acetaminophen (APAP) and dioctyl sodium sulfosuccinate (AOT) were purchased from Sigma-Aldrich (St. Louis, MO, USA). Chitosan (CS) with a degree of deacetylation of approximately $85 \%$ and molecular weight $250 \mathrm{kDa}$ was purchased from Koyo Chemical Co. Ltd. (Tokyo, Japan). Poly(acrylic acid) (PAA, Mv 450,000 g. $\mathrm{mol}^{-1}$ and $\mathrm{Mw} 1800 \mathrm{~g} \cdot \mathrm{mol}^{-1}$ was purchased from Sigma-Aldrich (St. Louis, MO, USA). Hydroxypropyl methylcellulose (HPMC) E3 was obtained from the Dow Chemical Company (Midland, MI, USA).

The chemical structure of the materials is given in Figure 1.

2.2. Solution of CS Films Preparation. CS was dissolved in a $1.5 \mathrm{M}$ of citric acid solution at a concentration of $2 \%$ (wt/vol) concentration. Films made by the solution were uniform and equivalent according to the AFM characterization.

2.3. Sample Preparation. Additive/APAP mixtures were prepared at $5 \%$ additive by first melting the mixture above the melting point and subsequently quenching the molten mixture in liquid nitrogen. The resultant solid was ground by a mortar and pestle for five minutes and followed by 15 minutes milling in a cryogenic mill (6750 freezer mill, Spex Sampleprep, Metuchen, NJ) to ensure complete mixing.

2.4. Crystal Growth Rate. The chitosan solution prepared in Section 2.2 was spin-coated on cover slides using a KW-4A spin coater (Chemat Technology Inc., Northridge, CA) at a speed of $2,000 \mathrm{rpm}$ and then dried in a desiccator to prepare the films.
The isothermal crystal growth rate of additive/APAP at $40^{\circ} \mathrm{C}$ on CS substrates was determined and performed by hot stage microscopy [16]. 3-5 mg of additive/APAP mixture was placed between two cover slips, melted at $185^{\circ} \mathrm{C}$, and subsequently quenched in liquid nitrogen. The samples were then examined using a polarizing microscope (Nikon Eclipse E600 POL microscope, Nikon Corp, Tokyo, Japan). The temperature of the sample was controlled by a hot stage (Linkam THMS600, Surrey, UK). As shown in Figure 2, the crystal of APAP grew as a spherulite. The pictures of the growing spherulite were taken at constant intervals using time-lapse photography. When the radius of the spherulite was plotted against time, it resulted in a linear line $\left(R^{2}>0.99\right)$, the slope of which was the growth rate.

2.5. Time-Resolved Wide-Angle X-Ray Scattering. The timeresolved WAXS experiments were conducted to study crystallization kinetics of additive/APAP on different substrates. The data were collected at the Advanced Photon Source Beam Station 12-ID-B, Argonne National Laboratory. The WAXS system was equipped with a Pilatus 300 detector. The energy of the X-ray source was $13.9984 \mathrm{keV}(\lambda$ $=0.88 \AA$ ), and the distance between the sample to the detector was $455.26 \mathrm{~mm}$. The $q$ (scattering vector) range was $0.9-2.12 \AA^{-1}$. The WAXS range was calibrated using silver behenate (AgBeh), and the absolute intensity was calibrated using glassy carbon.

$400 \mu \mathrm{l}$ of chitosan solution prepared in Section 2.2 was poured into small aluminum pans (Tzero DSC sample pans, TA Instruments, New Castle, DE) to prepare the chitosan films. The additive/APAP mixture was melted in a syringe by a syringe heater (Braintree Scientific, Inc., Braintree, MA), and $0.1 \mathrm{~mL}$ of molten additive/APAP was deposited on the film by a syringe pump, and the sample was quenched in liquid nitrogen. Subsequently, the pan was mounted on a Linkam THMS600 stage to control the crystallization temperature at $40^{\circ} \mathrm{C}$, and the WAXS patterns were collected at intervals of 5 seconds.

2.6. Thermal Analysis. At the crystallization temperature, $40^{\circ} \mathrm{C}$, which is much lower than the melting point of APAP, crystal growth of APAP is under diffusion control [17]. Therefore, the molecular mobility of APAP is one of the important keys that could affect crystallization. Here, thermal analysis was conducted to determine the glass transition temperatures $\left(T_{\mathrm{g}}\right)$ of APAP, and the value is used to evaluate the mobility of APAP in each system.

The physical mixture of 5\% wt/wt additive/APAP was analyzed by a differential scanning calorimeter (DSC). The thermal analysis was carried out using a TA Q100 DSC equipped with a refrigerated cooling accessory (TA Instruments, New Castle, DE). The instrument was calibrated for enthalpy and temperature using indium. $50 \mathrm{~mL} / \mathrm{min}$ of nitrogen was used as the purge gas. 3-5 mg of each sample was sealed into hermetic aluminum Tzero sample pans (TA instruments, New Castle, DE). $T_{\mathrm{g}}$ of APAP and additive/APAP mixtures were determined by cooling the melt at $20^{\circ} \mathrm{C} / \mathrm{min}$ to $-20^{\circ} \mathrm{C}$ and then reheating at $10^{\circ} \mathrm{C} / \mathrm{min}$. 
<smiles>CC(=O)Nc1ccc(O)cc1</smiles>

(a)<smiles>CCCCC(CC)COC(=O)CC(C(=O)OCC(CC)CCCC)S(=O)(=O)O[NH3+]</smiles>

(c)

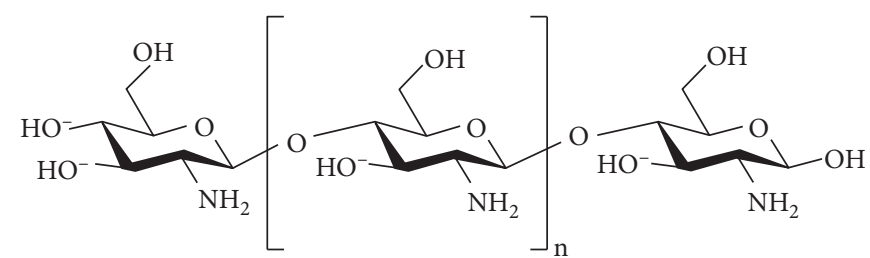

(b)

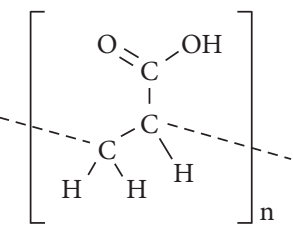

(d)

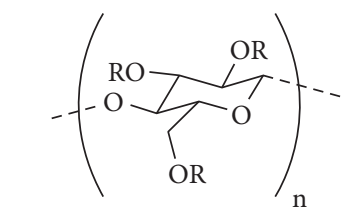

$\mathrm{R}=\mathrm{H}$ or $\mathrm{CH}_{3}$ or $\mathrm{CH}_{2} \mathrm{CH}(\mathrm{OH}) \mathrm{CH}_{3}$

(e)

Figure 1: Molecular structures of (a) APAP, (b) CS, (c) AOT, (d) PAA, and (e) HPMC.

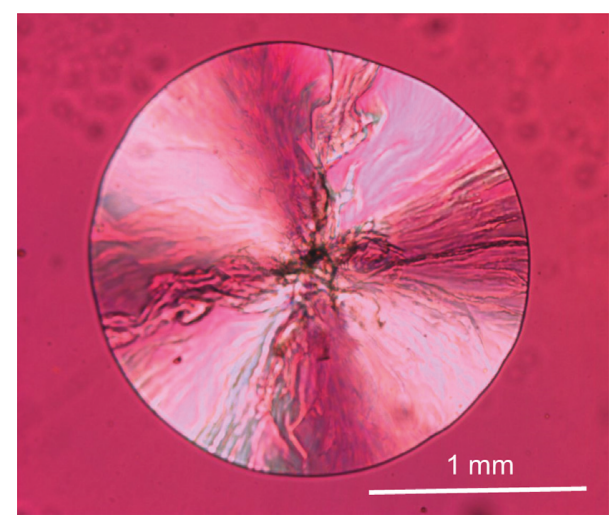

FIgURE 2: Photomicrographs of APAP crystal taken at $40^{\circ} \mathrm{C}$. The scale bar represents $1 \mathrm{~mm}$.

2.7. Fourier Transform Infrared (FTIR) Spectroscopy. FTIR was used to investigate interfacial interaction between the CS substrate and additive/APAP [16]. It was known that around $20-50 \% \mathrm{w} / \mathrm{w}$ additive is necessary to detect noticeable shifts in the infrared spectra arising from intermolecular interactions [18]. Therefore, solutions consisting of $30 \% \mathrm{wt} / \mathrm{wt}$ additive and $70 \% \mathrm{wt} / \mathrm{wt}$ APAP were prepared by dissolving the components in ethanol.

The samples were spin-coated onto ZnSe discs using a KW-4A spin coater (Chemat Technology Inc., Northridge, CA) set at a speed of 2,000 rpm. Spectra of pure APAP, CS substrate, pure additive, additive/APAP, and additive/APAP on CS were collected by a Nicolet Protégé 460 Fourier transform infrared spectrometer, equipped with a mercurycadmium-telluride (MCT) detector cooled with liquid nitrogen, in transmission mode. The spectral contributions from water vapor and carbon dioxide were minimized by continuously purging the instrument's sample chamber with dry air from a Balston purge gas generator. All spectra were taken at an interval of about $1 \mathrm{~cm}^{-1}$ with Happ-Genzel apodization and averaged over 256 scans.

\section{Results and Discussion}

3.1. Effect of Additives on Crystal Growth Rate of APAP. APAP is known to have three polymorphs [15]. Under physical constraint such as between two cover slips, melted APAP tends to yield form III [19]. This was confirmed by characterizing APAP that crystallized between two CScoated quartz cover slips via Raman spectroscopy.

Compared with samples without additives, from Figure 3, AOT, PAA, and HPMC were effective at decreasing the growth rate. AOT and HPMC are the least and most effective additives, respectively.

The effect of surfactants on crystal growth of celecoxib has been reported by Mosquera-Giraldo et al. [20]. Surfactants decreased $T_{\mathrm{g}}$ of celecoxib and increased its mobility and crystal growth rate. Nevertheless, in our study, AOT decreased the growth rate of APAP on CS substrates. In the experiments, the AOT/APAP mixture was sandwiched between the films; therefore, the impact of the films on crystal growth should be considered. The IR spectra of AOT-APAP-citric CS systems (Figure 4), show that the $\mathrm{N}-\mathrm{H}$ and $\mathrm{C}=\mathrm{O}$ stretch of APAP, and the combined $\mathrm{C}=\mathrm{O}$ stretch of AOT and APAP shifted to lower wavenumbers, indicating the presence of the CS film interfered with the binary interaction between AOT and APAP. AOT is an anionic surfactant, and it can have strong interactions with CS, which is a cationic polymer. As deposited on the CS film, the electrostatic interaction between AOT and 


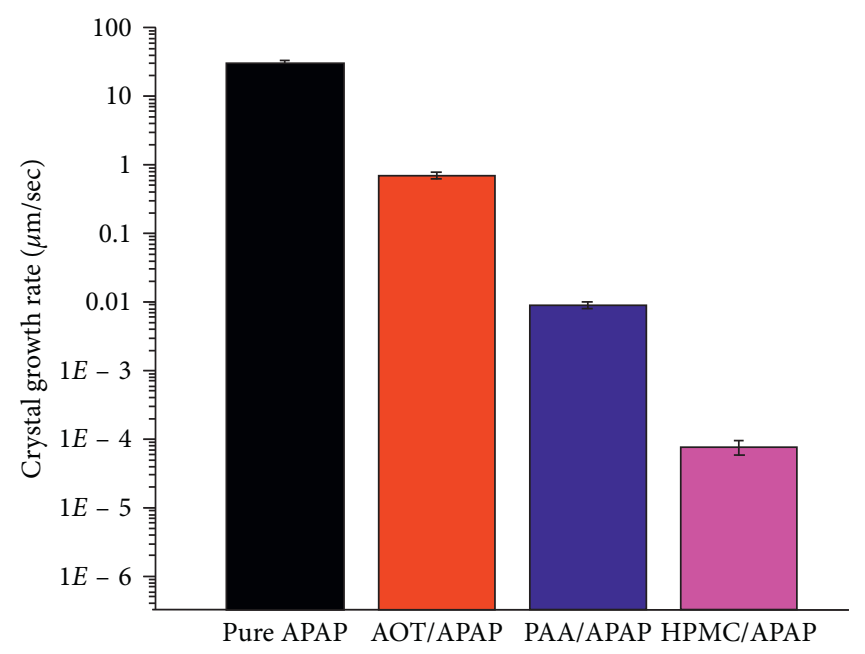

FIgURE 3: Interfacial crystal growth rate of APAP on citric CS films in the presence of different additives.

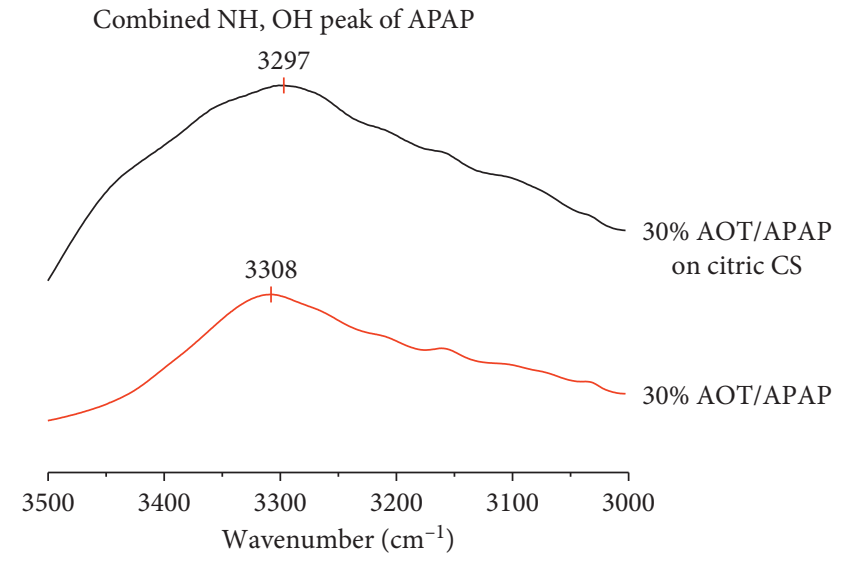

(a)

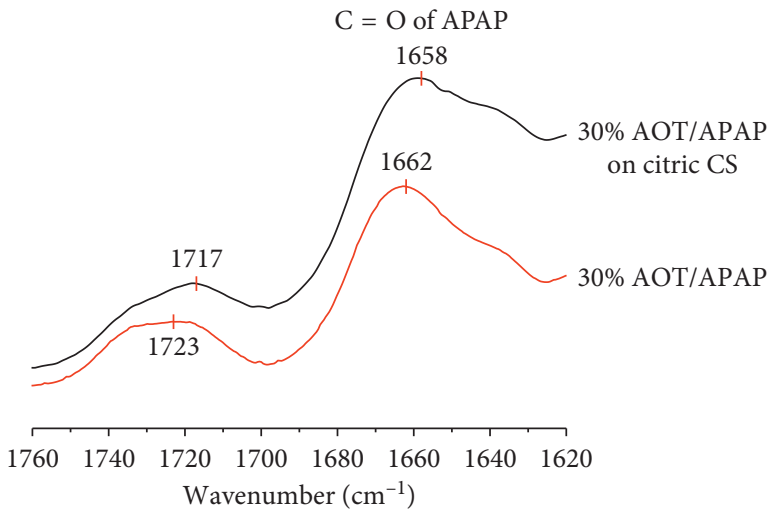

(b)

FIgURE 4: IR spectra of the AOT-APAP-citric CS system in the (a) combined N-H and O-H stretch region of APAP and (b) combined C=O stretch region of $\mathrm{AOT}$ and citric acid and $\mathrm{C}=\mathrm{O}$ stretch region of APAP.

CS could promote the phase separation of the AOT/APAP mixture. So, the faster mobility resulted by the surfactant is not expected and the increased growth rate was not observed. Besides, the CS film was found to increase the growth rate of APAP (paper under review). When blending, AOT can block some sites on the surface of the CS films that APAP can interact with, so the growth rate was slower compared with pure APAP grown on the CS film.

Previously, among a series of hydrophilic polymers, in the polymer-APAP binary system in the absence of the substrates, PAA was found to have the greatest effect on reducing the growth rate of APAP, whereas the impact from HPMC was less [18]. The stronger PAA-APAP molecular interaction was claimed to be the cause of the higher effectiveness. However, in the ternary additive-APAP-substrate system, the additive can interact with both APAP and the substrate. Similar to the AOT system, when blended, PAA can interact with APAP through $\mathrm{H}$-bonding. Besides, it can also form H-bonding between the carboxylic dimer and citric molecules doped on the CS film. Figure 5 shows the IR spectra of the $\mathrm{N}-\mathrm{H}, \mathrm{O}-\mathrm{H}$, and $\mathrm{C}=\mathrm{O}$ stretching regions of the samples containing $\mathrm{PAA}$ on citric the CS film. It can be seen that the functional groups all shifted to a lower wavenumber when the PAA/APAP mixture was coated on citric CS. Evidently, PAA in the blend was found to interact with the substrates, and the PAAsubstrate interaction can weaken the binary PAA-APAP interaction and decreases the effectiveness of PAA at decreasing the growth rate. On the contrary, as Figure 6 shows, the peaks in the HPMC-APAP-CS systems did not have noticeable shifts, indicating HPMC does not interact with the substrate much. The previous report also pointed out that the interaction between HPMC and APAP was very weak [18]. Here is a possible explanation for the slow growth rate when blending APAP with HPMC: at $40^{\circ} \mathrm{C}$, the additives are not miscible with APAP, and the additive-rich phase can also act like a substrate and affect crystallization of APAP. To confirm this hypothesis, the interfacial growth rate of APAP was characterized on PAA and HPMC films (Figure 7), and it was found when growing on HPMC film, the crystal growth rate was much lower compared with on glass or PAA film. 


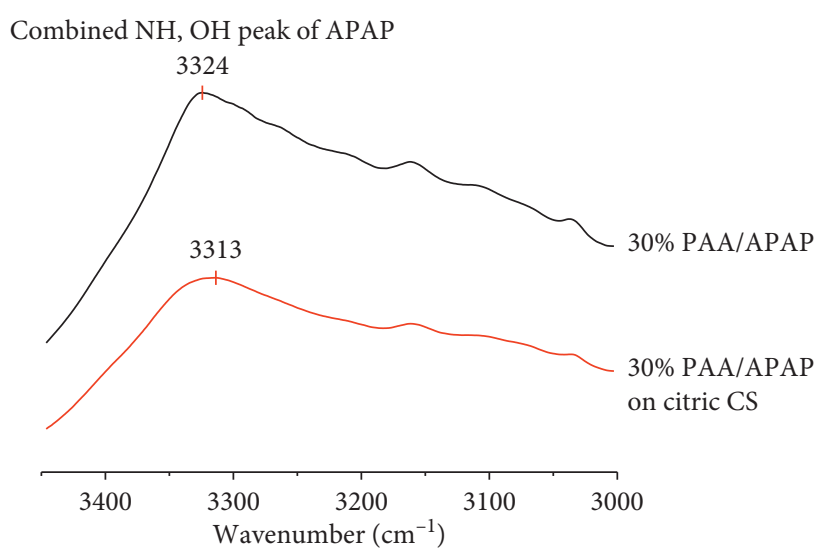

(a)

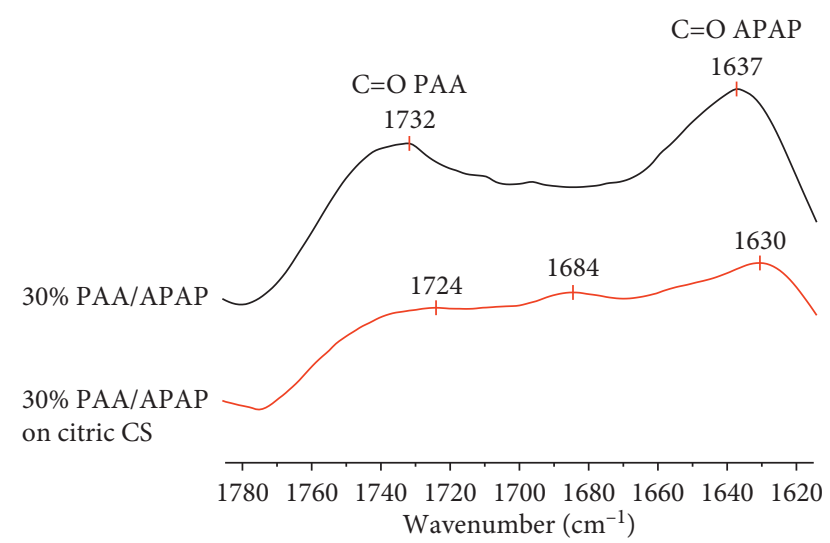

(b)

FIGURE 5: IR spectra of the PAA-APAP-citric CS system in the (a) combined N-H and O-H stretch region of APAP and (b) combined C=O stretch region of PAA and citric acid and $\mathrm{C}=\mathrm{O}$ stretch region of APAP.

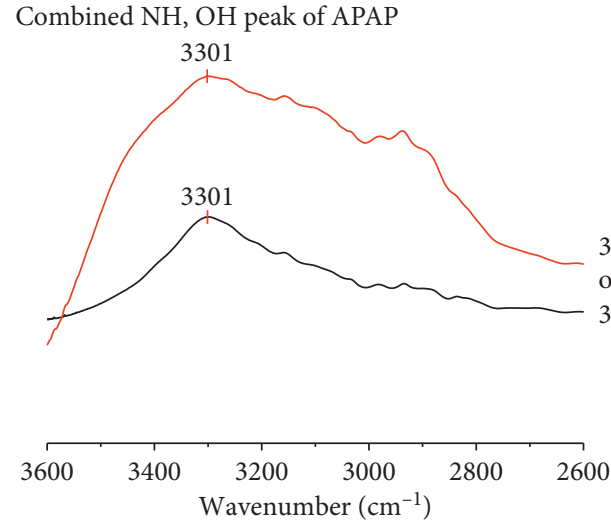

(a)
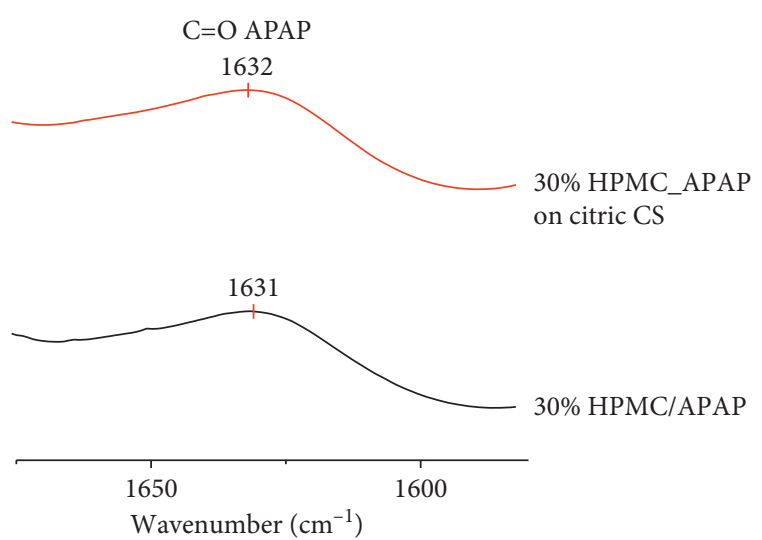

(b)

FIgURE 6: IR spectra of the HPMC-APAP-citric CS system in the (a) combined N-H and O-H stretch region of APAP and (b) C=O stretch region of citric acid and APAP.

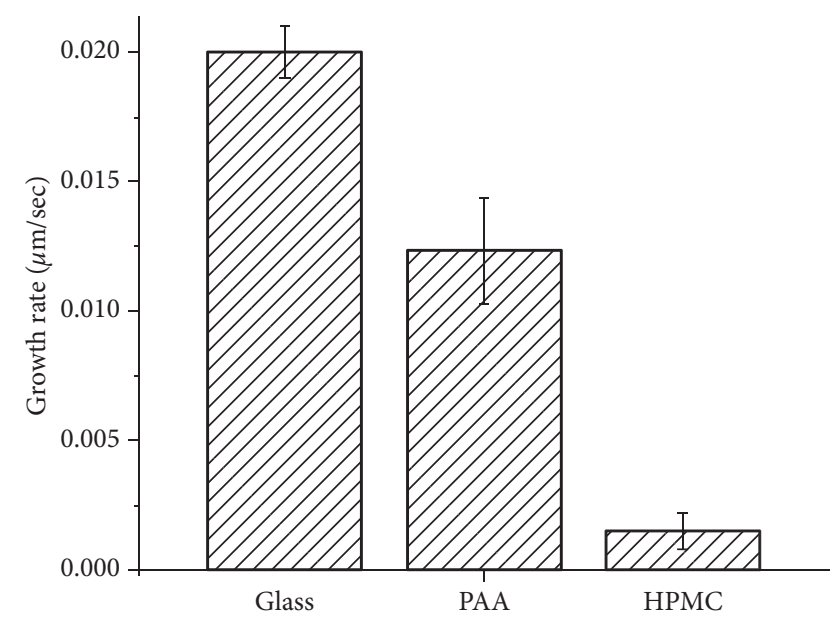

FIGURE 7: Interfacial crystal growth rate of APAP on glass, PAA, and HPMC films.
3.2. Effects of Additives on Crystallization Kinetics of APAP. In addition to the interfacial study of crystal growth rate, the isothermal crystallization kinetics of additive/APAP on the CS film without confinement was also investigated using the time-resolved WAXS at the Argonne National Lab. The dynamic WAXS patterns of the samples containing additives and CS substrate were collected, and it was observed that APAP crystallized into Form I.

The WAXS patterns of AOT/APAP on CS at different time points are shown in Figure 8. Initially, the small peaks appeared at 0 second were contributed by the aluminum pan, and peaks corresponded to crystalline APAP shown up at about 1 minute. The percentage of crystalline APAP at different time periods, denoted as $X(t)$, was calculated by integrating the area under the APAP diffraction peaks at time $t\left(\phi_{\mathrm{APAP}}(t)\right)$ and normalized by the area under the APAP diffraction peaks when crystallization was complete $\left(\phi_{\mathrm{APAP}}(\infty)\right)[4,12,21-24]$. 


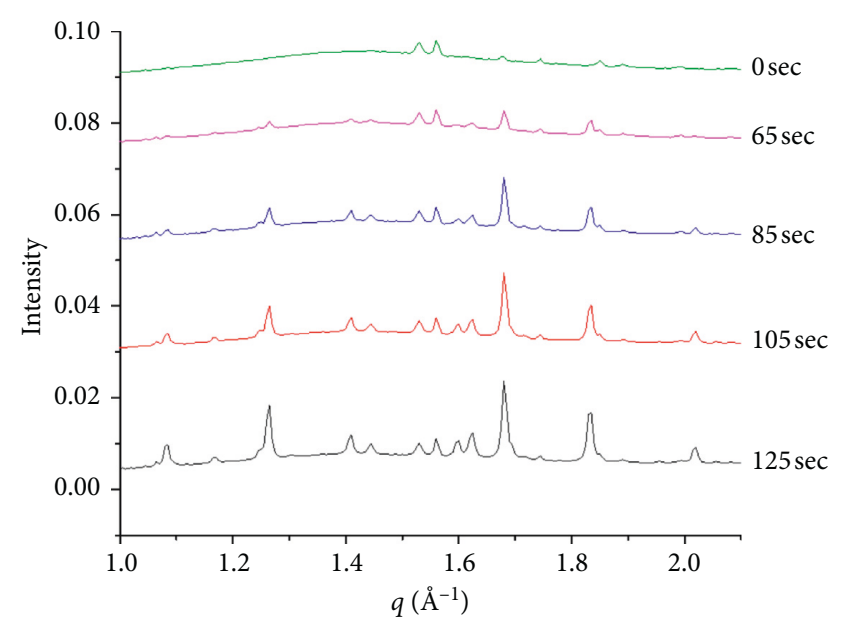

FIgURE 8: WAXS patterns of 5\% AOT/APAP on the CS film at different time points.

$$
X(t)=\frac{\phi_{\mathrm{APAP}}(t)}{\phi_{\mathrm{APAP}}(\infty)}
$$

The induction time $\left(t_{\text {in }}\right)$ and the time until $50 \%$ of the sample crystallized $\left(t_{50}\right)$ for each sample are summarized in Table 1. HPMC was found to increase the induction time, $t_{\mathrm{in}}$, the most compared with AOT and PAA, which is consistent with the previous study, which showed that HPMC was much more effective at inhibiting nucleation of APAP than PAA [18]. Samples containing PAA and HPMC had longer $50 \%$ crystallization times, $t_{50}$, compared with pure APAP on CS substrates.

In contrast to the interfacial growth rate study, the observed trend of $t_{50}$ is consistent with the observed binary additive-APAP systems which were published $[18,20]$. AOT decreased $t_{50}$ of the samples. From the thermal analysis of additive/APAP mixture (Table 2), AOT decreased $T_{\mathrm{g}}$ of APAP, and therefore, the increased mobility was suspected to be the reason for the shorter $t_{50}$ for AOT-added samples. PAA and HPMC both extended $t_{50}$, and the impact of PAA was stronger than HPMC.

Without confinement, only additives close to the interface can be affected by the substrate, and the influence was not large enough to affect the additive-APAP interaction in the bulk. It is likely that when allowed to crystallize in three dimensions, the additive-APAP interaction is more significant at controlling crystallization of APAP. Therefore, to confirm this hypothesis, crystal growth rate of APAP with additive and on different CS films was characterized without being covered by the top layer. In our previous research (paper under review), CS films prepared by different acids can have a profound impact on the crystallization of APAP. However, as Figure 9 demonstrates, when growing without confinement, the effect of substrates was negligible, and the impact of additives was consistent with the observed binary additive-APAP systems. AOT was found to increase the growth rate, and PAA had a stronger effect at reducing the growth rate than HPMC. This confirmed that additiveAPAP interactions are more important at determining the
TABLE 1: Summary of crystallization parameters of each system on CS films.

\begin{tabular}{lccccccc}
\hline $\begin{array}{l}\text { Pure } \\
\text { APAP }\end{array}$ & \multicolumn{2}{c}{ AOT/ } & \multicolumn{2}{c}{ PAA } & \multicolumn{2}{c}{ HPMC/ } \\
\hline$t_{\text {in }}$ & $t_{50}{ }^{*}$ & $t_{\text {in }}$ & $t_{50}{ }^{*}$ & $t_{\text {in }}$ & $t_{50}{ }^{*}$ & $t_{\text {in }}$ & $t_{50}{ }^{*}$ \\
1 & 1.5 & 1 & 0.5 & 5 & 9.57 & 30 & 7.76 \\
\hline${ }^{*} t_{\text {in }}$ is not included in & $t_{50}{ }^{* *}$ The unit is minute. & &
\end{tabular}

TABLE 2: Glass transition temperature $\left(T_{\mathrm{g}}\right)$ of $5 \%$ additive/APAP.

\begin{tabular}{ccccc}
\hline & APAP & AOT/APAP & PAA/APAP & HPMC/APAP \\
\hline$T_{\mathrm{g}}\left({ }^{\circ} \mathrm{C}\right)$ & $23.9 \pm 0.2$ & $19.3 \pm 0.4$ & $25.4 \pm 0.3$ & $24.6 \pm 0.4$ \\
\hline
\end{tabular}

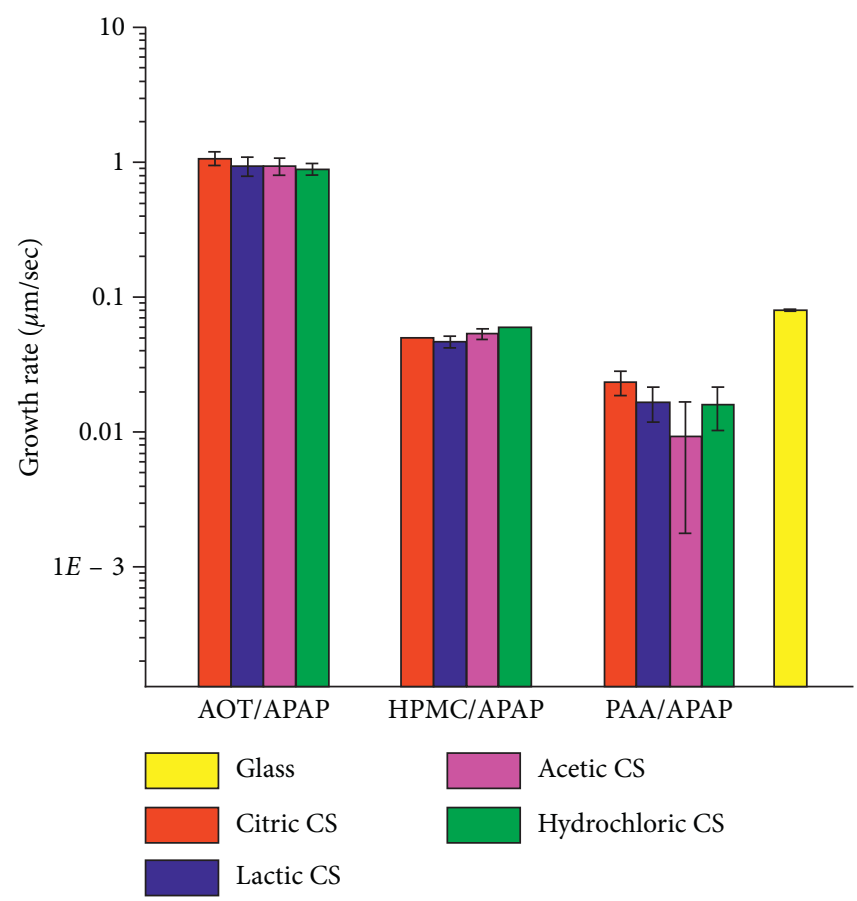

Figure 9: Growth rate of 5\% additive/APAP on CS films without being sandwiched.

crystallization of APAP when it is allowed to crystallize in three dimensions.

\section{Conclusion}

With the presence of three different additives, the crystallization behavior of APAP on the CS film was characterized. In the study of interfacial crystal growth rate, the strong additive-substrate interaction can interfere with the interactions between the additive and APAP and change the crystallization behavior observed in the binary additiveAPAP system. On the contrary, when allowed to crystallize in three dimensions, the additive-APAP interaction is more significant in controlling the crystallization of APAP. To conclude, when study crystallization at the interface, additive-substrate interaction can be important, and it can weaken the additive-APAP interaction; whereas, without 
confinement, the additive-APAP interaction is more significant at controlling crystallization.

\section{Data Availability}

The data used to support the findings of this study are available from the corresponding author upon request.

\section{Conflicts of Interest}

The authors declare that they have no conflicts of interest.

\section{References}

[1] P. Debenedetti, Metastable Liquids: Concepts and Principles, Princeton University, Princeton, NJ, USA, 1996.

[2] P. W. Carter and M. D. Ward, "Topographically directed nucleation of organic crystals on molecular single-crystal substrates," Journal of the American Chemical Society, vol. 115, no. 24, pp. 11521-11535, 1993.

[3] Y. Diao, A. S. Myerson, T. A. Hatton, and B. L. Trout, "Surface design for controlled crystallization: the role of surface chemistry and nanoscale pores in heterogeneous nucleation," Langmuir, vol. 27, no. 9, pp. 5324-5334, 2011.

[4] J. L. Quon, K. Chadwick, G. P. F. Wood et al., “Templated nucleation of acetaminophen on spherical excipient agglomerates," Langmuir, vol. 29, no. 10, pp. 3292-3300, 2013.

[5] A. L. Grzesiak and A. J. Matzger, "New form discovery for the analgesics flurbiprofen and sulindac facilitated by polymer-induced heteronucleation," Journal of Pharmaceutical Sciences, vol. 96, no. 11, pp. 2978-2986, 2007.

[6] L. M. Foroughi, Y. N. Kang, and A. J. Matzger, "Polymerinduced heteronucleation for protein single crystal growth: structural elucidation of bovine liver catalase and concanavalin a forms," Crystal Growth \& Design, vol. 11, no. 4, pp. 1294-1298, 2011.

[7] I. Weissbuch, R. Popovitz-Biro, M. Lahav, L. Leiserowitz, and Rehovot, "Understanding and control of nucleation, growth, habit, dissolution and structure of two-and three-dimensional crystals using tailor-made auxiliaries," Acta Crystallographica Section B Structural Science, vol. 51, no. 2, pp. 115-148, 1995.

[8] P. Hartman and W. G. Perdok, "On the relations between structure and morphology of crystals," Acta Crystallographica, vol. 8, no. 1, pp. 49-52, 1955.

[9] D. M. Walba, H. A. Razavi, N. A. Clark, and D. S. Parmar, "Design and synthesis of new ferroelectric liquid crystals. 5. Properties of some chiral fluorinated FLCs: a direct connection between macroscopic properties and absolute configuration in a fluid phase," Journal of the American Chemical Society, vol. 110, no. 26, pp. 8686-8691, 1988.

[10] L. J. W. Shimon, M. Vaida, L. Addadi, M. Lahav, and L. Leiserowitz, "Molecular recognition at the solid-solution interface: a relay mechanism for the effect of solvent on crystal growth and dissolution," Journal of the American Chemical Society, vol. 112, no. 17, pp. 6215-6220, 1990.

[11] I. Weissbuch, D. Zbaida, L. Addadi, L. Leiserowitz, and M. Lahav, "Design of polymeric inhibitors for the control of crystal polymorphism. Induced enantiomeric resolution at racemic histidine by crystallization at 25 . Degree. C," Journal of the American Chemical Society, vol. 109, no. 6, pp. 1869$1871,1987$.

[12] E. M. Landau, S. G. Wolf, M. Levanon, L. Leiserowitz, M. Lahav, and J. Sagiv, "Stereochemical studies in crystal nucleation. Oriented crystal growth of glycine at interfaces covered with Langmuir and Langmuir-Blodgett films of resolved. alpha.-amino acids," Journal of the American Chemical Society, vol. 111, no. 4, pp. 1436-1445, 1989.

[13] I. Weissbuch, F. Frolow, L. Addadi, M. Lahav, and L. Leiserowitz, "Oriented crystallization as a tool for detecting ordered aggregates of water-soluble hydrophobic. alpha.-amino acids at the air-solution interface," Journal of the American Chemical Society, vol. 112, no. 21, pp. 7718-7724, 1990.

[14] S. Mann, B. Heywood, S. Rajam, and V. Wade, "Molecular Recognition in Biomineralization," in Proceedings of Mechanisms and Phylogeny of Mineralization in Biological Systems, pp. 47-55, Springer, Tokyo, Japan, 1991.

[15] M. L. Peterson, S. L. Morissette, C. McNulty et al., "Iterative high-throughput polymorphism studies on acetaminophen and an experimentally derived structure for form III," Journal of the American Chemical Society, vol. 124, no. 37, pp. 10958-10959, 2002.

[16] H. Hsu, O. O. Adigun, L. S. Taylor, S. Murad, and M. T. Harris, "Crystallization of acetaminophen on chitosan films blended with different acids," Chemical Engineering Science, vol. 126, pp. 1-9, 2015.

[17] V. Andronis and G. Zografi, "Crystal nucleation and growth of indomethacin poly-morphs from the amorphous state," Journal of Non-Crystalline Solids Solids, vol. 271, no. 3, pp. 236-248, 2000.

[18] N. S. Trasi and L. S. Taylor, "Effect of polymers on nucleation and crystal growth of amorphous acetaminophen," CrystEngComm, vol. 14, no. 16, pp. 5188-5197, 2012.

[19] A. L. Grzesiak and A. J. Matzger, "Selection and discovery of polymorphs of platinum complexes facilitated by polymerinduced heteronucleation," Inorganic Chemistry, vol. 46, no. 2, pp. 453-457, 2007.

[20] L. I. Mosquera-Giraldo, N. S. Trasi, and L. S. Taylor, "Impact of surfactants on the crystal growth of amorphous celecoxib," International Journal of Pharmaceutics, vol. 461, no. 1-2, pp. 251-257, 2014.

[21] M. S. Lisowski, Q. Liu, J. Cho, J. Runt, F. Yeh, and B. S. Hsiao, "Crystallization behavior of poly (ethylene oxide) and its blends using time-resolved wide-and small-angle X-ray scattering," Macromolecules, vol. 33, no. 13, pp. 4842-4849, 2000 .

[22] F. J. Baltá-Calleja and C. G. Vonk, X-Ray Scattering of Synthetic Polymers, Elsevier Science, New York, NY, USA, 1989.

[23] J. C. Burley, M. J. Duer, R. S. Stein, and R. M. Vrcelj, "Enforcing Ostwald's rule of stages: isolation of paracetamol forms III and II," European Journal of Pharmaceutical Sciences, vol. 31, no. 5, pp. 271-276, 2007.

[24] Q. Zhu, S. J. Toth, G. J. Simpson, H. Y. Hsu, L. S. Taylor, and M. T. Harris, "Crystallization and dissolution behavior of naproxen/polyethylene glycol solid dispersions," Journal of Physical Chemistry B, vol. 117, no. 5, pp. 1494-1500, 2013. 


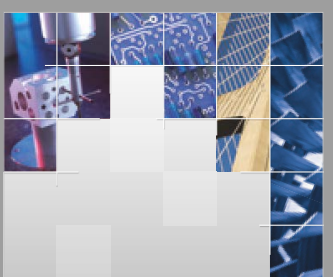

\section{Enfincering}
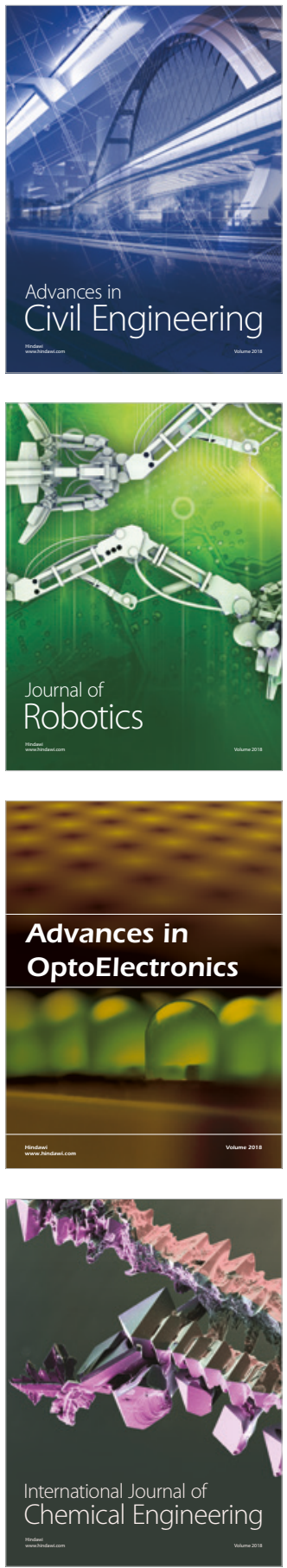

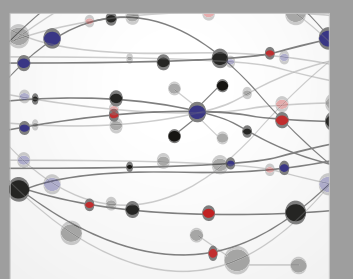

\section{Rotating \\ Machinery}

The Scientific World Journal

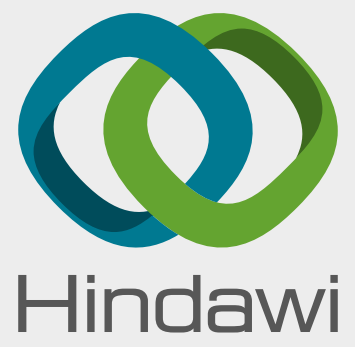

Submit your manuscripts at

www.hindawi.com
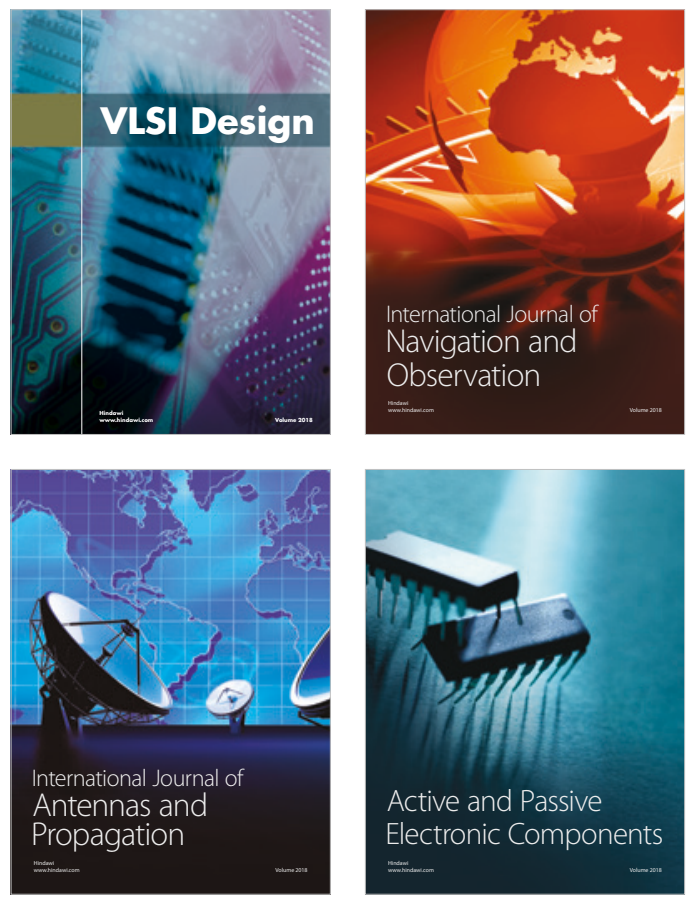
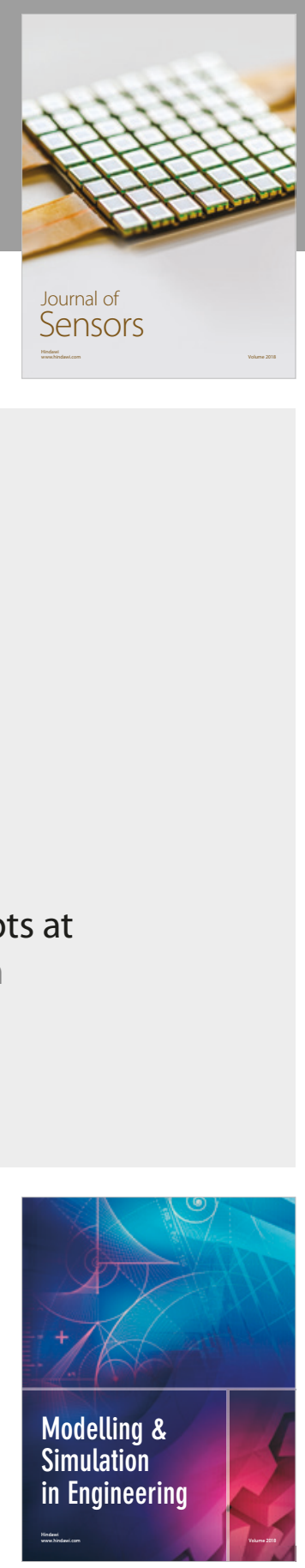

\section{Advances \\ Multimedia}
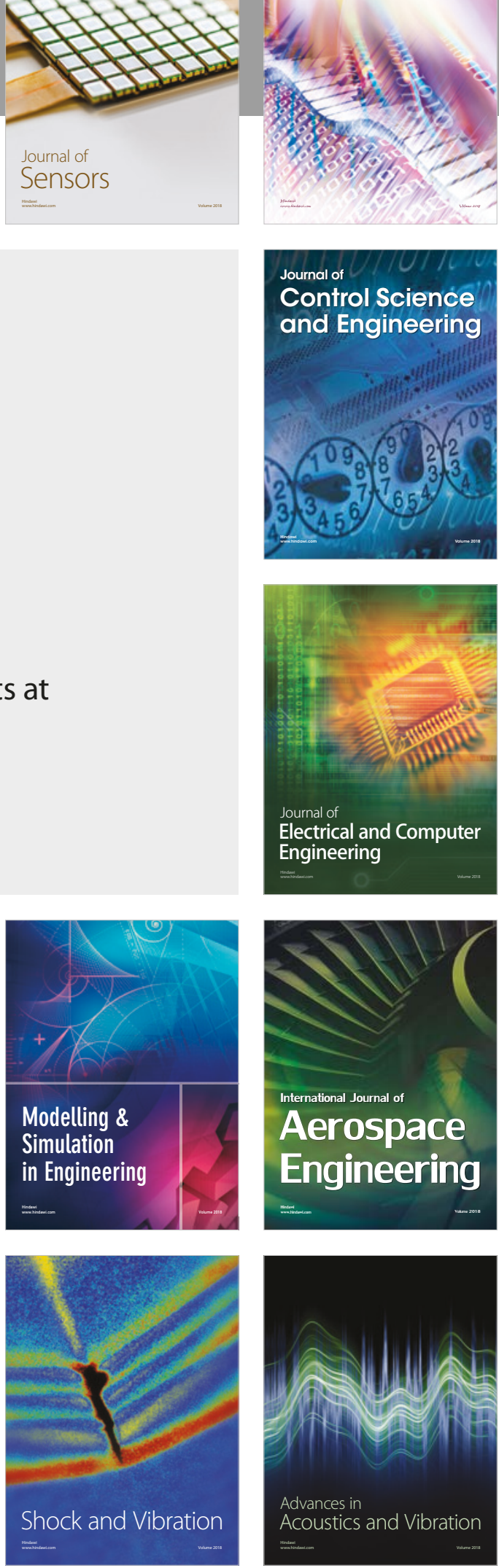